\title{
Algas alimenticias para mejorar la calidad nutritiva de los productos cárnicos
}

\section{Food algae to improve the nutritional quality of meat products}

Brita Anaya González' , Elbert Hermoza Valdivia²

Universidad Nacional San Cristóbal de Huamanga

\section{RESUMEN}

Objetivos: Mejorar la calidad nutritiva de los productos cárnicos a través de algas alimenticias. Métodos: Investigación básica experimental, con un diseño aleatorizado. La población estuvo constituida por diversos tipos de carne: res, cordero, cerdo y alpaca; y algas: Nostoc sp. conocido como nostoc, Gigartina chamissoi (qochayuyo) y Ulva sp. (yuyo) que llegan al mercado Nery García de la ciudad de Huamanga, capital del departamento de Ayacucho. La muestra comprendió $2 \mathrm{~kg}$ de cada tipo de carne y algas. La determinación de nutrientes fue sobre la base de los métodos de la AOAC (Official Methods of Analysis). Resultados: Al ser comparadas 5 mezclas de diferentes proporciones de carnes y algas, la diferencia encontrada fue significativa entre el contenido de valor calórico $(P=0,000)$ y nutritivo $(P=0,000)$. La mezcla $A$ fue la ideal con un porcentaje de proteínas de 31,87 $\mathrm{g} \%$, las grasas con un valor de $12,95 \mathrm{~g} \%$, inferior a lo existente en las carnes lo que es favorable para una disminución de riesgos de enfermedades. El porcentaje de carbohidratos reportó $7,10 \mathrm{~g} \%$ cantidad baja, pero con buen tenor de fibra 16,00 $\mathrm{g} \%$ sumamente importante para el peristaltismo y buen funcionamiento del sistema digestivo. La cantidad de cenizas de 4,02 $\mathrm{g} \%$, significa que existe un buen aporte de minerales indispensables para el organismo. Conclusiones: Se mejoró la calidad nutritiva de los productos cárnicos al adicionar algas, con un valor calórico de $272,43 \mathrm{kcal} / 100 \mathrm{~g}$ satisfactorio, y el valor nutritivo de 2,41 lo identifica como altamente nutritivo.

Palabras clave: Algas alimenticias, calidad nutritiva, productos cárnicos.

$1 \mathrm{Mg}$. Docente Principal e investigadora de la Universidad Nacional San Cristóbal de Huamanga.

2 Blgo. Docente Asociada e investigador de la Universidad Nacional San Cristóbal de Huamanga 


\section{ABSTRACT}

Objectives: To improve the nutritional quality of meat products through food algae. Methods: Experimental Basic research, with a randomized design. The population was formed by various meat types: beef, lamb, pork and alpaca; and algae: Nostoc sp. known as nostoc, Gigartina chamissoi (qochayuyo) and Ulva sp. (yuyo) that arrive in the Nery García market from Huamanga, Ayacucho department's capital. The sample was formed by $2 \mathrm{~kg}$ of each meat and algae type. Nutrient determination was based on the AOAC (Official Methods of Analysis methods. Results: 5 mixtures of different meat and algae proportions were compared, the found difference was significant between caloric value content $(P=0,000)$ and nutritional $(P=0,000)$. Mixture $A$ was the ideal with a protein percentage of $31,87 \mathrm{~g} \%$, fats with a value of $12,95 \mathrm{~g} \%$, lower than what meat have which is favorable for a disease risk reduction. The carbohydrates percentage is $7,10 \mathrm{~g} \%$ low amount, but with good fiber tenor of 16,00 $\mathrm{g} \%$, extremely important for peristalsis and the digestive system proper functioning. The ash quantity of $4,02 \mathrm{~g} \%$ means that there is a good essential minerals supply for the body. Conclusions: Nutritional quality of meat products were improved by adding algae, with a satisfactory caloric value of $272,43 \mathrm{kcal} / 100 \mathrm{~g}$, and the nutritional value of 2,41 identifies it as highly nutritious.

Keywords: Food algae, nutritional quality, meat products.

\section{INTRODUCCIÓN}

Hoy cobra importancia el uso de los alimentos funcionales en la dieta diaria. Esta investigación trata de incorporar las algas a los alimentos cárnicos para enriquecer su calidad nutricional con alimentos de origen vegetal, desde un punto de vista tecnológico y nutracéutico, y así darle a estos ingredientes un uso más, en la alimentación regional.

El empleo de las algas alimenticias, se considera una estrategia clave en el sector alimentario, y presenta un impacto directo en la generación de las características organolépticas y físico químicas, de los diferentes productos cárnicos; es significativa la proporción de alimentos procesados en la dieta diaria que se van incrementando continuamente, la tendencia actual es la alimentación vegetariana, ya que dietas ricas en carnes van a ocasionar diversos trastornos cardiovasculares (1).

Araya considera recomendable incrementar la concentración de un componente natural del alimento para alcanzar una concentración que se espera que induzca los efectos deseados, por ejemplo, agregar un componente que no está normalmente presente en la mayor parte de los alimentos, para el cual se haya demostrado efectos beneficiosos (fito-químicos y antioxidantes), y reemplazar un componente del alimento, generalmente un macronutriente cuya ingesta sea excesiva y que muestre efectos deletéreos, como el reemplazo de grasa por fibra dietética, componente beneficioso para la salud (1).

Tabacchi y Osborne, refieren que el contenido de humedad en el análisis de carnes, está en función de la gordura de la carne $(2,3)$. Por esta razón, Sumar plantea la difusión del consumo de nuestros productos nativos como las carnes de alpaca y llama, que tienen menor contenido graso (4).

Acleto y Sumarriva, por su parte, analizaron la composición química de diversas algas marinas y de agua dulce, reportando un alto contenido proteico $(5,6)$.

El problema planteado en la investigación fue: ìmejorará la calidad nutritiva de los productos cárnicos al adicionar algas alimenticias?

Los objetivos fueron, determinar los componentes químico bromatológicos de las carnes, algas y de la mezcla; y mejorar la calidad nutritiva de los productos cárnicos a través de algas alimenticias.

La hipótesis fue: la calidad nutritiva de los 
productos cárnicos, mejora al adicionar algas alimenticias.

El aporte de la investigación radica en que la mezcla elaborada proporciona una mejora sustancial de proteínas con aminoácidos de alta calidad; y el contenido de grasas es inferior respecto a los niveles en las carnes, por lo que la presencia de grasas saturadas es menor, lo que resulta favorable para la salud de las personas de la comunidad en general.

La limitación en el estudio estuvo en la adquisición de las muestras, que son sólo de temporada en caso de las algas, y en caso del nostoc su procedencia alto andina.

\section{MATERIAL Y MÉTODOS}

La población estuvo constituida por los diversos tipos de carne: res, cordero, cerdo y alpaca; y por las algas: Nostoc sp. (nostoc), Gigartina chamissoi (qochayuyo) y Ulva sp. (yuyo) que llegan al mercado Nery García de la ciudad de Huamanga capital del departamento de Ayacucho.

La muestra comprendió $2 \mathrm{~kg}$ de cada tipo de carne y algas, productos que fueron trasladados al laboratorio de bioquímica para su secado total y obtención de harinas, para los respectivos análisis químico bromatológicos en el laboratorio de bromatología de la Facultad de Ciencias Biológicas de la Universidad Nacional de San Cristóbal de Huamanga.

Para la recolección de muestras se tomó en cuenta las recomendaciones de la AOAC (Official Methods of Analysis) (7), los productos comestibles y los ingredientes de alimentos son materiales muy heterogéneos, por lo que es difícil obtener una sola muestra absolutamente representativa; por dificultades prácticas y económicas el muestreo fue realizado al azar.

Los métodos utilizados para averiguar el contenido de nutrientes, tanto en los productos al estado inicial, como después de haber efectuado la adición de las algas alimenticias, fueron en base a los establecidos por la AOAC (7), como son: determinación de humedad o contenido acuoso por el método de secado; cenizas por incineración; proteínas por micro Kjeldahl, el extracto etéreo, a través del método de Soxhlet; fibra, por hidrólisis ácida y alcalina; carbohidratos totales, mediante el empleo de la diferencia de 100 con respecto a la suma de todos los demás nutrientes (proteínas, grasas, ceniza); cuantificación del valor nutritivo, basada en la fórmula de Atwater (8) y para el valor calórico, se ha hecho uso de los valores promedios por gramo de cada uno de los macronutrientes.

El estudio comprendió cinco formulaciones (Mezcla A, B, C, D y E) con diferentes proporciones de carnes y algas.

La investigación fue básica experimental. Se realizó el análisis de varianza (ANVA) y las comparaciones múltiples de los promedios con la prueba de Tukey.

\section{RESULTADOS}

Los resultados reflejan los valores promedios de los análisis químicos trabajados por triplicado en cada caso, la determinación inicial fue de cada muestra (tabla 1 y 2 ).

Se ha realizado cinco formulaciones con

Tabla N ${ }^{\circ}$ 1: Porcentaje del análisis químico de las carnes.

\begin{tabular}{lccccc}
\hline & $\begin{array}{c}\text { Humedad } \\
\mathbf{g} \%\end{array}$ & $\begin{array}{c}\text { Ceniza } \\
\mathbf{g} \%\end{array}$ & $\begin{array}{c}\text { Proteína } \\
\mathbf{g} \%\end{array}$ & $\begin{array}{c}\text { Lípido } \\
\mathbf{g} \%\end{array}$ & $\begin{array}{c}\text { Carbohidrato } \\
\mathbf{g} \%\end{array}$ \\
\hline Cerdo & 59,00 & 1,19 & 37,80 & 22,05 & 37,80 \\
Res & 69,00 & 0,91 & 42,14 & 26,74 & 42,14 \\
Alpaca & 66,15 & 1,80 & 40,65 & 23,52 & 40,65 \\
Cordero & 71,71 & 2,68 & 20,36 & 5,78 & 20,36 \\
\hline
\end{tabular}


Tabla N²: Porcentaje del análisis químico de las algas.

\begin{tabular}{lcccccc}
\hline & $\begin{array}{c}\text { Humedad } \\
\mathrm{g} \%\end{array}$ & $\begin{array}{c}\text { Ceniza } \\
\mathbf{g} \%\end{array}$ & $\begin{array}{c}\text { Proteína } \\
\mathbf{g} \%\end{array}$ & $\begin{array}{c}\text { Lípido } \\
\mathbf{g} \%\end{array}$ & $\begin{array}{c}\text { Carbohidrato } \\
\mathbf{g} \%\end{array}$ & Fibra g\% \\
\hline Qochayuyo & 14,00 & 2,02 & 14,18 & 4,09 & 13,92 & 51,80 \\
Yuyo & 80,84 & 16,28 & 41,14 & 1,20 & 19,75 & 21,63 \\
Nostoc & 83,17 & 4,62 & 13,12 & 1,02 & 9,91 & 47,99 \\
\hline
\end{tabular}

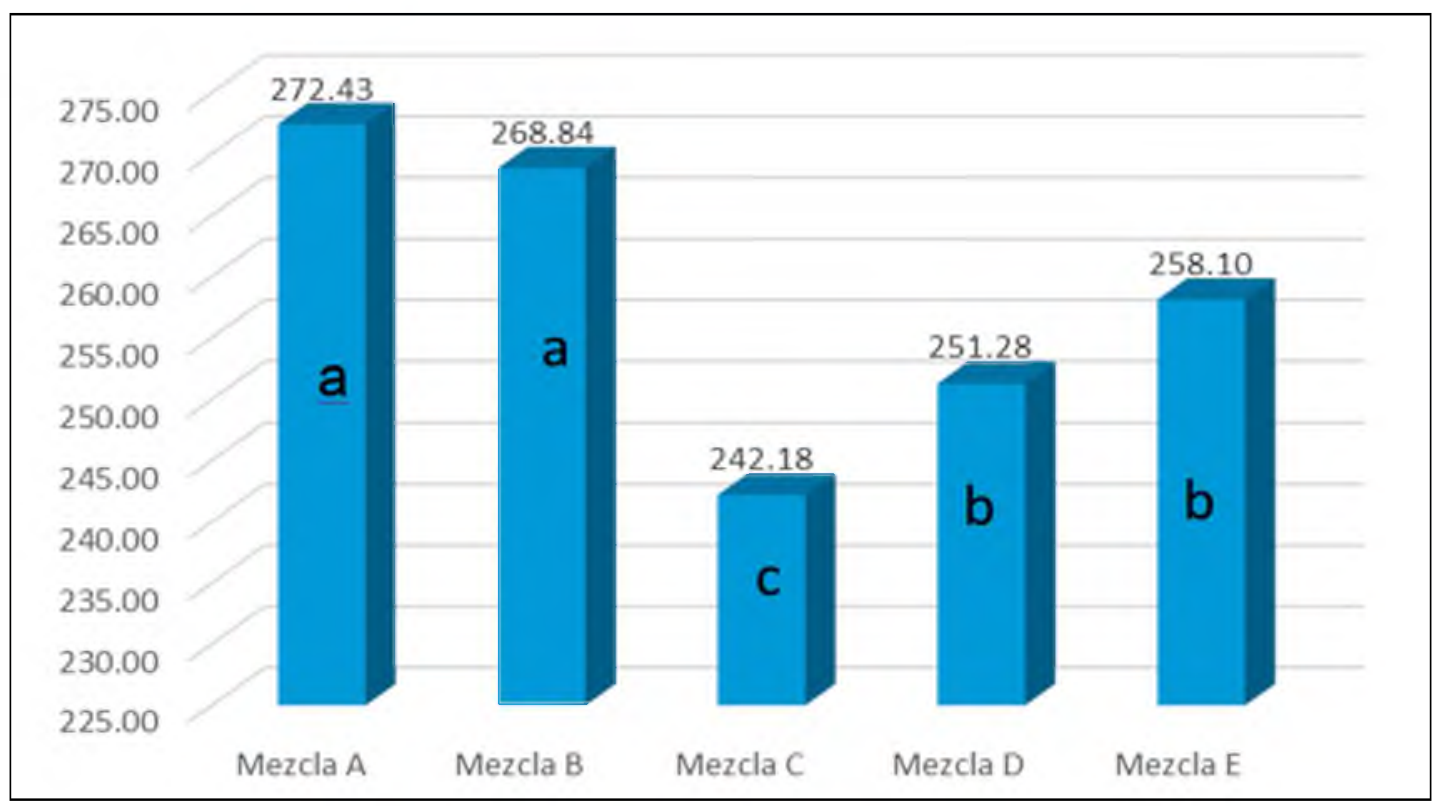

Figura $\mathrm{N}^{\circ}$ 1: Promedios de valor calórico de las cinco mezclas.

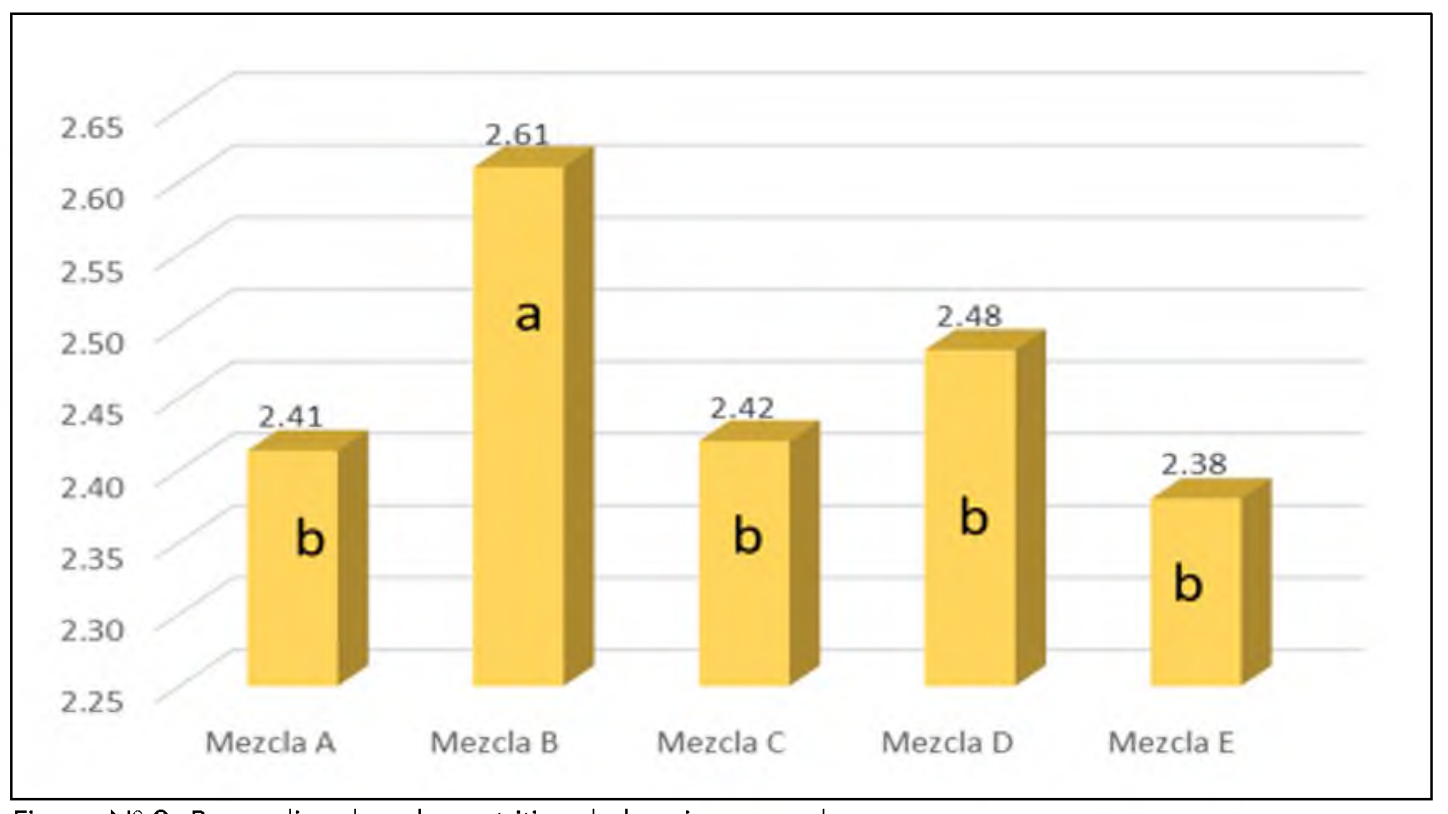

Figura $\mathrm{N}^{\circ}$ 2: Promedios de valor nutritivo de las cinco mezclas. 
Tabla N 3: Porcentaje del análisis químico de la mezcla A (ideal) de carnes y algas.

\begin{tabular}{lcccccc}
\hline & $\begin{array}{c}\text { Porcentaje } \\
\mathbf{g} \%\end{array}$ & $\begin{array}{c}\text { Ceniza } \\
\mathbf{g} \%\end{array}$ & $\begin{array}{c}\text { Proteína } \\
\mathbf{g} \%\end{array}$ & $\begin{array}{c}\text { Lípido } \\
\mathbf{g} \%\end{array}$ & $\begin{array}{c}\text { Carbohidrato } \\
\mathbf{g} \%\end{array}$ & $\begin{array}{c}\text { Fibra } \\
\mathbf{g} \%\end{array}$ \\
\hline Cerdo & 15,00 & 0,18 & 5,67 & 3,31 & 0,00 & 0,00 \\
Alpaca & 20,00 & 0,36 & 8,13 & 4,70 & 0,00 & 0,00 \\
Res & 15,00 & 0,14 & 6,36 & 4,01 & 0,00 & 0,00 \\
Cordero & 10,00 & 0,27 & 2,04 & 0,58 & 0,00 & 0,00 \\
Qochayuyo & 20,00 & 0,40 & 2,84 & 0,82 & 2,78 & 10,36 \\
Yuyo & 15,00 & 2,44 & 6,17 & 0,18 & 2,96 & 3,24 \\
Nostoc & 5,00 & 0,23 & 0,66 & 0,05 & 1,36 & 2,40 \\
Total & 100,00 & 4,02 & 31,87 & 12,95 & 7,10 & 16,00 \\
\hline
\end{tabular}

tres repeticiones, el análisis de varianza y las comparaciones múltiples de los promedios con la prueba de Tukey se muestran en las figuras 1 y 2 , cuyo resultado arroja como ideal la mezcla A. (tabla 3)

Valor calórico de la mezcla A con los resultados de la tabla 3 .

Proteínas: $\quad 31,87 \times 4=127,48$

Lípidos: $\quad 12,95 \times 9=116,55$

Carbohidratos: $7,10 \times 4=28,40$

$=272,43 \mathrm{Kcal} / 100 \mathrm{~g}$

Valor nutritivo de la mezcla A.

$V N=\frac{2,40 \times 116,55+28,40}{127,48}=2,41$

\section{DISCUSIÓN}

El contenido de nutrientes en las carnes, está en función al tipo de animal, tipo de alimentación, la etapa fisiológica, entre otros factores, así a menor edad, contiene más agua, es más suave y digerible; a mayor edad, tiene más grasa y más sabor (9).

Del análisis químico bromatológico respecto a la carne de cerdo utilizada se califica como semigrasa, con valor promedio de 59 $\mathrm{g} \%$; Tabacchi (2), reporta que el cerdo con carne magra, tiene $68,5 \mathrm{~g} \%$ de humedad, superior al hallado en el trabajo, lo que indica que la carne utilizada en el trabajo es de mayor gordura; Osborne (3) señala un 59,84 $\mathrm{g} \%$ de humedad, similar a lo encontrado en el trabajo; en consecuencia el contenido de humedad está en función a la gordura de la carne.

La carne de res por las características organolépticas que presenta es considerada semigrasa, con $69 \mathrm{~g} \%$ de humedad; según Osborne (3), el contenido acuoso es de 74,26 $\mathrm{g} \%$, ligeramente superior a lo reportado en el trabajo. Tabacchi (2), indica que la carne de res tiene un $71 \mathrm{~g} \%$ concordante con lo hallado en el trabajo; asimismo, los valores reportados por los diferentes autores son ligeramente superiores a los nuestros, lo que significa que las carnes son menos gordas que la del trabajo.

En cuanto a la carne de alpaca, el valor promedio del contenido de humedad fue $66,15 \mathrm{~g} \%$; Sumar (4), reporta valores que varían de 71,90 a $77,30 \mathrm{~g} \%$, cifras superiores a lo reportado, lo que nos indica que la carne de alpaca con la que se trabajó es de mayor gordura.

El contenido de humedad en la carne de cordero presenta una media de 71,71 g\%, según Osborne (3) la humedad de la carne de ovino es de 73,12 g\% similar a lo reportado, es decir que son animales con las mismas características y condiciones al momento de haber sido sacrificados.

Respecto al porcentaje de cenizas en los diferentes tipos de carnes, se observa que la de res tiene menor contenido $(0,91 \mathrm{~g} \%)$ que la de cordero $(2,68 \mathrm{~g} \%)$, que coincide con lo reportado por Sumar $(2,16 \mathrm{~g} \%)(4)$, 
con lo que podemos decir que los animales utilizados tienen las mismas condiciones de gordura; en caso de una carne de cerdo gorda, los minerales se encuentran concentrados.

El porcentaje de proteínas encontrado en la carne de cerdo fue de $37,80 \mathrm{~g} \%$, Tabacchi (2), refiere que la carne magra de cerdo tiene 18,5 g\% y Osborne (3) reporta 21,01 $\mathrm{g} \%$ inferior a lo hallado en el trabajo, debido a que la carne trabajada fue de mayor gordura, con menor cantidad de líquidos motivo que hace que la cantidad de proteínas sea mayor.

Los valores de proteínas en carne de res son en base a materia seca, que oscilan entre 37,8 y $45,58 \mathrm{~g} \%$, Osborne (3) reporta $23,43 \mathrm{~g} \%$, lo que indica que trabajó en materia fresca; por otra parte Tabacchi (2), señala $20,0 \mathrm{~g} \%$ en carne magra y $16,5 \mathrm{~g} \%$ en carne gorda, que también significa que usó muestra fresca.

En carne de alpaca se encontró un valor promedio de 40,65 $\mathrm{g} \%$ de proteínas, mientras, que Osborne (3), reporta valores entre 18,93 a $21,70 \mathrm{~g} \%$, lo que indica que la carne con que se trabajó es más gorda que la otra. Así mismo Sumar (4), manifiesta que la cantidad de proteínas que tiene la carne de alpaca es de $21,93 \mathrm{~g} \%$ inferior al obtenido en la investigación.

El promedio de proteínas en la carne de cordero oscila entre 19,80 y $20,93 \mathrm{~g} \%$ y una media de 20,36 g \%, Osborne (3) reporta $18,19 \mathrm{~g} \%$, semejante a lo hallado en el trabajo, lo que significa que al momento del sacrificio los animales tenían las mismas condiciones de gordura.

Respecto al contenido de lípidos es mayor en la carne de res $(26,74 \mathrm{~g} \%)$ seguida de la alpaca y cerdo, y el menor valor en la carne de cordero $(5,78 \mathrm{~g} \%)$; en caso de la carne de cerdo, ésta es de mayor gordura, pues al comparar con los valores hallados por otros autores los valores son menores: Tabacchi $11,9 \mathrm{~g} \%$, Osborne 4,84 g\%, en este caso se trata de una carne totalmente magra $(2,3)$. En general la grasa de res, cerdo, alpaca y cordero tiene más ácidos grasos saturados que insaturados y colesterol. Sus triglicéridos son de iguales o distintos ácidos grasos, traducidos en suavidad, sabor, color y olor propio (9). Al adicionar algas a las carnes disminuye el contenido lipídico (en especial las grasas saturadas) como se muestra en la tabla 3, lo que significa reducir los riesgos que causan las enfermedades coronarias, arterioesclerosis, hipercolesterolemia, al consumir sólo carnes.

El valor de lípidos en la carne de res comparado a los hallados por Tabacchi $(6,5 \mathrm{~g} \%)$ y Osborne $(4,84 \mathrm{~g} \%)$ es mayor, lo que quiere decir que la carne con la que se trabajó presenta mayor gordura y la de los otros autores es totalmente magra $(2,3)$.

Para el caso de las algas, el contenido de humedad del "qochayuyo" es de $14 \mathrm{~g} \%$, comparado con las referencias resulta menor porque el análisis lo hicieron en base fresca con una cifra de $81 \mathrm{~g} \%$ (5).

El porcentaje de humedad del "yuyo" es de $79,45 \mathrm{~g} \%$; mientras que Acleto (5), reporta un valor de $81,32 \mathrm{~g} \%$ bastante próximo al encontrado en el trabajo, en caso del "nostoc" arroja un promedio de 6,44 g\% que comparada a la de otras algas es inferior.

El contenido de cenizas en el "qochayuyo" es de 2,02 $\mathrm{g} \%$, inferior al resto, en cambio el "yuyo" presenta 16,28 g\%, que guarda coincidencia con lo reportado por Acleto (5) de $15,61 \mathrm{~g} \%$. El "nostoc" presenta un valor intermedio de $4,62 \mathrm{~g} \%$.

En cuanto a la cuantificación de proteínas en el "qochayuyo", se obtuvo 14,18 g\% como promedio, ligeramente superior a lo señalado por Sumarriva (8) 11,26 g\%. Para el "yuyo" se reporta una cifra de 40,12 g\%, similar al obtenido por Acleto (5) $(42,92$ $\mathrm{g} \%)$. En caso del "nostoc" el valor fue menor al resto, es decir 13,12 g\%.

Las algas en cuanto al contenido de lípidos que en realidad se expresa como extracto etéreo, presentan valores bajos; el "qochayuyo" alcanza una cifra de 4,09 g\%, 
superior a lo reportado por Sumarriva $(0,27$ $\mathrm{g} \%$ ) (6). El "yuyo" alcanza valores de 1,2 $\mathrm{g} \%$; Acleto reporta un valor de $0,123 \mathrm{~g} \%$ inferior a lo reportado en el trabajo, para "nostoc" se reporta un valor de 1,06 g\% similar a lo hallado por Acleto. (5)

El porcentaje de fibra obtenido en las algas, para el "qochayuyo" arroja un promedio de $51,80 \mathrm{~g} \%$, bastante similar a lo reportado por Sumarriva $(47,5 \mathrm{~g} \%)$ (6) La fibra del "yuyo" alcanza un valor de 21,63 g\%, lo que indica que es un buen alimento como para que exista un normal tránsito intestinal. En los trabajos consultados se reportan como carbohidratos totales con los que existe cierta similitud. En el "nostoc" la fibra presenta un valor de 47,99 $\mathrm{g} \%$ parecido al "qochayuyo". Las fibras son moléculas parcialmente digeridas por los microorganismos del colon, donde se hidratan y se mezclan con las heces, forman un bolo fecal blando, fácil de evacuar y aceleran los movimientos peristálticos, lo que evita que las heces sean secas, duras y que puedan concentrar toxinas que permanezcan en contacto con la mucosa intestinal (9).

El contenido de carbohidratos obtenido por diferencia de 100 frente a la suma de los otros nutrientes, para el "qochayuyo" muestra un valor de $12,98 \mathrm{~g} \%$, frente al dato reportado por Sumarriva (6), de 8,67 g\%; en caso del "yuyo" se reporta 19,75 $\mathrm{g} \%$, Acleto (5) refiere una cifra mayor $(41,34 \mathrm{~g} \%)$.

Al hacer las comparaciones de las cinco formulaciones, resulta adecuada la mezcla A de la tabla 3 , se aprecia que la cantidad de proteínas es de 31,87 g\%, lo que es más importante es haber realizado una mezcla en la que existen proteínas con aminoácidos de alta calidad; en cuanto a grasas, se tiene el valor de $12,95 \mathrm{~g} \%$ que resulta inferior a lo existente en las carnes que alcanza hasta $26 \mathrm{~g} \%$; lo que es favorable para la salud, debido a la menor cantidad de ácidos grasos saturados, colesterol y triacilgliceroles que contiene normalmente los diversos tipos de carne.
Los alimentos de origen vegetal, además de nutrir, dan bienestar físico y mental, reducen riesgos de enfermedad, previenen o retrasan trastornos o enfermedades (9); al disminuir el contenido de lípidos en el preparado, más la mezcla con las algas, estaría actuando como alimento funcional. En cuanto a los carbohidratos se reporta $7,10 \mathrm{~g} \%$ constituyendo una cantidad baja, pero existe buen tenor de fibra 16,00 g\% que es sumamente importante para el peristaltismo y buen funcionamiento del sistema digestivo; la cantidad de cenizas es de 4,02 $\mathrm{g} \%$, con lo que existe un buen aporte de minerales que son indispensables para el organismo.

Según Araya, es recomendable incrementar la concentración de un componente natural del alimento para alcanzar una concentración que se espera que induzca los efectos deseados, por ejemplo, la fortificación con micronutrientes para lograr una ingesta mayor que las recomendaciones dietéticas, compatible con los valores sugeridos para disminución de riesgos de enfermedades, agregar un componente que no está normalmente presente en la mayor parte de los alimentos, para el cual se haya demostrado efectos beneficiosos (fitoquímicos y antioxidantes), y reemplazar un componente del alimento, generalmente un macronutriente cuya ingesta sea excesiva y que muestre efectos deletéreos, por ejemplo el reemplazo de grasa por fibra dietética, componente beneficioso para la salud (1); al combinar las carnes con las algas también se está aportando fibra que resulta benéfico para la salud.

Producto de la combinación de cinco tipos de mezclas, se obtiene como mejor la mezcla A (tabla 3); existe una diferencia significativa entre las medias del valor calórico y nutritivo (figuras 1 y 2), la mezcla A proporciona un valor calórico de 272,43 Kcal/100g valorado como satisfactorio y mayor que las otras mezclas; el valor nutritivo de 2,41 lo ubica como altamente nutritivo de carácter proteico. De acuerdo a la fórmula de Atwather, cifras del valor nutritivo menores a 3,8 se considera como altamente proteico, en cambio cifras mayores a 3,8 representan 
alimentos energéticos (3). Si bien la mezcla B tiene mayor valor nutritivo, debido al gran porcentaje de carne de alpaca presente; sin embargo, el valor calórico es menor, además hay que tomar muy en cuenta el olor no muy agradable de este tipo de carne; asimismo el contenido en grasas es mayor que en la mezcla $A$.

El valor nutritivo de los productos cárnicos es muy bien conocido. Su salubridad puede ser mejorada, equilibrando su relación omega-6/omega-3. De forma natural, la mayoría de las carnes poseen una relación omega-6/omega-3 superior a 15. (10) La estrategia más favorable para reducir dicho valor es incorporar a los productos cárnicos ácidos grasos omega-3 y esto se estaría logrando al incorporar alimentos vegetales (algas).

Se concluye que mejoró la calidad nutritiva de los productos cárnicos al adicionar algas, con un valor calórico de 272,43 kcal/100 g satisfactorio, y el valor nutritivo de 2,41 lo identifica como altamente nutritivo.

\section{REFERENCIAS BIBLIOGRÁFICAS}

1. Araya $\mathrm{H}$, Lutz M. Alimentos funcionales y saludables. Rev. chil. nutr. 2003; 30(1): 8-14.

2. Tabacchi P, García F. Caracterización bioquímica del alga gigartina chamissoi (Bahía de Ancón). Revista de Química. 1994; 8(1): $21-28$.

3. Osborne $R$, Voot $P$ Análisis de los nutrientes de los alimentos. Zaragoza: Acribia S.A; 2005.

4. Sumar J. Llamas y alpacas. Universidad de Chile: Facultad de Ciencias veterinarias y pecuarias; 2008.

5. Acleto C. Algas marinas del Perú de importancia económica. Lima: Universidad Nacional Mayor de San Marcos. Museo de Historia Natural; 1996.

6. Sumarriva L. Estudio de la composición química de algunas algas de mayor consumo en el Perú. Lima: Alfaomega; 1985.

7. AOAC. Official Methods of Analysis. Washington D.C.: Association of Official Agricultural Chemists; 2004.

8. Paredes C. Nutrición. Fundamentos bioquímicos, fisiológicos y clínicos. Lima: Grafimag S.R.L; 1993.

9. Blanco T. Alimentación y nutrición. Lima: Lettera Gráfica S.A.; 2011.

10. Palanca $V$, Rodríguez $E$, Señoráns J, Reglero G. Scientific bases for the development of functional meat products with combined biological activity. Nutr Hosp. 2006; $21(2)$ : 199202. 